\title{
Silvia Lorusso, Le Charme sans la beauté, vie de Sophie Cottin
}

\section{Gabriella Bosco}

\section{(2) OpenEdition}

1 Journals

\section{Edizione digitale}

URL: https://journals.openedition.org/studifrancesi/21869

DOI: 10.4000/studifrancesi.21869

ISSN: 2421-5856

\section{Editore}

Rosenberg \& Sellier

\section{Edizione cartacea}

Data di pubblicazione: 1 décembre 2019

Paginazione: 623-624

ISSN: 0039-2944

\section{Notizia bibliografica digitale}

Gabriella Bosco, «Silvia Lorusso, Le Charme sans la beauté, vie de Sophie Cottin», Studi Francesi [Online] 189 (LXIII | III) | 2019, online dal 01 mars 2020, consultato il 11 novembre 2021. URL: http:// journals.openedition.org/studifrancesi/21869; DOI: https://doi.org/10.4000/studifrancesi.21869

Questo documento è stato generato automaticamente il 11 novembre 2021.

\section{(c) (i) $\odot$}

Studi Francesi è distribuita con Licenza Creative Commons Attribuzione - Non commerciale - Non opere derivate 4.0 Internazionale. 


\title{
Silvia Lorusso, Le Charme sans la beauté, vie de Sophie Cottin
}

\author{
Gabriella Bosco
}

\section{NOTIZIA}

Silvia Lorusso, Le Charme sans la beauté, vie de Sophie Cottin, Classiques Garnier, Paris, 2018, 356 pp.

1 Vissuta a cavallo tra Sette e Ottocento, Sophie Cottin ha attraversato vicende storiche e personali di natura tale da farla percepire più come personaggio leggendario che come persona reale. Pochi e in certi casi poco attendibili gli studi a lei consacrati sino alle soglie del xx secolo, e anche il Novecento si è rivelato parco di attenzione critica per vita e opere di colei cui Silvia Lorusso dedica invece ormai da tempo lavori di ricerca approfondita al fine di restituirle la dignità letteraria che ritiene spettarle.

2 Come alcuni dei suoi predecessori, e in particolare il più affidabile di loro, Leslie Clifford Sykes, autore nel 1949 di uno studio biografico su Sophie Cottin, Silvia Lorusso attinge alla ricca corrispondenza della donna, «source fondamentale» scrive nell'Avantpropos (pp. 13-16), «non seulement pour l'étude de l'œuvre de Mme Cottin, mais aussi pour l'histoire du roman au début du XIX ${ }^{\mathrm{e}}$ siècle». La figura che Silvia Lorusso tratteggia, grazie al suo poderoso volume, è quella quindi di una Sophie Cottin sottratta finalmente alle deformazioni consuete del biografismo romanzato tanto in auge nel secolo scorso, e restituita tramite un'attenta esegesi dei documenti presi in esame a un'attendibilità storica e letteraria che ne mette in luce la vitale tendenza alla contraddizione esistenziale: «écrivaine, mais hostile aux femmes auteurs; protestante, mais capable d'exalter la confession catholique; partisane du rôle domestique de la femme, masi aussi de son droit au divorce; heureuse dans son mariage, mais pessimiste dans ses romans sur l'amour conjugal; austère et coquette; passionnée et en même temps rationnelle; laide et d'un charme irrésistible...» (p. 14). Ecco alcune delle motivazioni che hanno certamente contribuito a destare l'interesse della studiosa nei 
confronti di Sophie Cottin, unitamente a quella principale che già altrove Silvia Lorusso ha interpretato come un réfoulement della sua opera, con conseguente espulsione dal canone romanzesco, dovuto in gran parte al suo coinvolgimento nei fatti rivoluzionari e alla loro violenza.

La ricostruzione della vicenda biografica di Sophie Cottin prende così le mosse a partire da luogo e data di nascita - Parigi, Place des Victoires, 22 marzo 1770 - attentamente ristabilita nonostante i dubbi emessi dalla critica precedente, sempre consultata e valutata da Silvia Lorusso sia negli aspetti positivi, i contributi documentati, che in quelli negativi, ovvero la frequente infondatezza delle informazioni. E procede poi dipanando una matassa di dati che incessantemente consentono al racconto via via costruito di far la spola tra la vita e l'opera di Sophie (così spesso Silvia Lorusso chiama la sua autrice, con il nome di battesimo, «sachant bien - scrive - que je m'autorise une liberté qui n'abolit pourtant ni mon respect, ni mon attitude critique», p. 15).

4 La biografia si struttura in numerosi capitoli che hanno il pregio della brevità relativa cui fa da contraltare la ricchezza di elementi forniti tramite, come si diceva, il costante ricorso alle lettere dell'autrice, sin da quelle degli anni giovanili dalle quali emerge la frequentazione degli scritti di Rousseau, autore che la madre di Sophie riteneva ipocrita e che lei invece, diciannovenne, già considerava il suo maître à penser $\mathrm{e}$ contemporaneamente il suo meilleur ami. Dall'infanzia al matrimonio con Paul Cottin, banchiere in Parigi, facoltoso e protestante, all'attraversamento della Rivoluzione da parte della giovane coppia, il viaggio in Inghilterra quando la situazione si complica. Poi il ritorno a Parigi, il Terrore, la morte di Paul, il lutto della giovane donna e il lento ritorno alla vita sociale in seguito a una fase di ritiro in campagna. Dopo tanti anni di letture da parte di Sophie, ecco il suo approdo alla scrittura. Centrale, il capitolo che Silvia Lorusso consacra ai maestri di Sophie (pp. 99-108) così come, parallelamente, quello sui suoi amori («Une femme fatale sans être belle», come già diceva di lei SainteBeuve, pp. 109-130). Trampolini, entrambi, per la parte del volume dedicata alla romanziera, che lascia spazio tuttavia a interessanti considerazioni relative ai dubbi di Sophie nei confronti di quell'attività, la scrittura, da parte di una donna, dubbi che convivono in lei sino alla fine («malgré moi ma tête compose toujours», avrebbe confessato a un'amica sul letto di morte, come testimonia una lettera inedita, cfr. $p$. 152) con la pratica della scrittura stessa. Claire d'Albe, Malvina, romanzi all'epoca di grande successo, cui faranno seguito prima Mathilde e poi Elisabeth ou les Exilés de Sibérie, il romanzo dell'emancipazione - scrive Silvia Lorusso - dallo stile dei precedenti, definito da Suard oriental, romanzo "presque sans amour». Vi è poi un capitolo sul viaggio in Italia di Sophie, effettuato nel 1806 per accompagnare la moglie di un cugino che soffriva di una malattia nervosa cui i medici avevano consigliato il Grand Tour a scopo terapeutico, racconto scandito dai reportages tratti dalla corrispondenza. Infine la morte, il 25 agosto 1807, con la ricostruzione delle controversie legate alla supposta conversione di Sophie al cattolicesimo.

5 Il volume comporta poi ancora un "état des lieux" riguardo agli inediti in poesia e in prosa conservati tra i manoscritti di Mme Cottin, così come quelli di alcune sue traduzioni. Quanto al testo intitolato L'Isola Bella attribuito in passato a Sophie Cottin, Silvia Lorusso argomenta con convinzione il proprio disaccordo. E conclude il volume un capitolo sulla fortuna, che sarebbe più corretto considerare la sfortuna, come viene ampiamente dimostrato, di Sophie Cottin dopo la morte, sfortuna cui secondo Benjamin Constant contribuirono la scarsa avvenenza e una vita amorosa postvedovile 
misteriosa, ma che viceversa non è sbagliato mettere in relazione con l'evolvere del giudizio critico di Stendhal nei suoi confronti, inizialmente positivo e poi via via sempre più negativo.

6 In conclusione tuttavia Silvia Lorusso dà conto di quella che può essere considerata, grazie al lavoro di un certo numero di storici della letteratura cui va oggi aggiunto il suo, un ritorno d'interesse per Sophie Cottin e gli aspetti indubbiamente trasgressivi della sua figura e della sua opera.

7 Apparati di grande spessore (note, annessi genealogici, bibliografia e indici) fanno di questo volume la migliore delle introduzioni per la pubblicazione delle Cuvres complètes di Sophie Cottin che usciranno prossimamente nei Classiques Garnier a cura della stessa Silvia Lorusso e di Fabienne Bercegol. 